Mens

revue d'histoire intellectuelle de l'Amérique française

\title{
Robert Rumilly, passeur des droites nationalistes entre la France et le Canada français
}

\section{Olivier Dard}

Volume 11, numéro 2, printemps 2011

URI : https://id.erudit.org/iderudit/1023371ar

DOI : https://doi.org/10.7202/1023371ar

Aller au sommaire du numéro

Éditeur(s)

Centre de recherche en civilisation canadienne-française

ISSN

1492-8647 (imprimé)

1927-9299 (numérique)

Découvrir la revue

Citer cet article

Dard, O. (2011). Robert Rumilly, passeur des droites nationalistes entre la France et le Canada français. Mens, 11(2), 7-36.

https://doi.org/10.7202/1023371ar
Résumé de l'article

Cet article s'attache à Robert Rumilly et à ses fonctions de passeur des droites nationalistes entre la France et le Canada français. Français marqué originellement par le maurrassisme et arrivé au Canada en 1928 pour en prendre la nationalité en 1934, Rumilly n'est jamais retourné en France. Le propos s'articule chronologiquement en trois étapes. Dans un premier temps, il s'agit d'analyser la relation entre Rumilly, le maurrassisme et le Canada français. L'article s'intéresse ensuite à l'épuration que ce pétainiste convaincu dénonce vivement au Canada en recyclant des thématiques venues de France. Ce recyclage s'accompagne, dans un troisième temps, d'un placage et d'une réinterprétation lorsque Rumilly stigmatise, sous le nom d'« infiltration gauchiste ", l'emprise des " catholiques de gauche » et la subversion à l'œuvre au Canada. 


\title{
Articles
}

\section{Robert Rumilly, passeur des droites nationalistes entre la France et le Canada français}

\author{
Olivier Dard \\ UFR sciences humaines et arts \\ Université Paul Verlaine - Metz
}

\section{Résumé}

Cet article s'attache à Robert Rumilly et à ses fonctions de passeur des droites nationalistes entre la France et le Canada français. Français marqué originellement par le maurrassisme et arrivé au Canada en 1928 pour en prendre la nationalité en 1934, Rumilly n'est jamais retourné en France. Le propos s'articule chronologiquement en trois étapes. Dans un premier temps, il s'agit d'analyser la relation entre Rumilly, le maurrassisme et le Canada français. L'article s'intéresse ensuite à l'épuration que ce pétainiste convaincu dénonce vivement au Canada en recyclant des thématiques venues de France. Ce recyclage s'accompagne, dans un troisième temps, d'un placage et d'une réinterprétation lorsque Rumilly stigmatise, sous le nom d' "infiltration gauchiste ", l'emprise des " catholiques de gauche » et la subversion à l'œuvre au Canada.

\section{Abstract}

This article focuses on Robert Rumilly and on his role as a transmitter between the right-wing nationalisms of France and French Canada. Born 
in France, Rumilly was strongly influenced by the thought of Charles Maurras. He immigrated to Canada in 1928, became a naturalised citizen in 1934, and would never again return to France. The present article will be elaborated chronologically and in three stages. Firstly, the relationship between Rumilly, maurrasism and French Canada will be examined. His denunciation of the purge that followed the collapse of the Vichy regime will then be analysed. Rumilly, a convinced Petainist, recycled French themes on the épuration in his Canadian writing. Finally, his Canadian crusade against "left-wing infiltration," subversion, and the influence of "left-wing Catholics" will be examined. In this postwar campaign, Rumilly's long-standing tendency to recycle French themes in his writing was accompanied by a corresponding tendency to reinterpret Canadian events in light of right-wing French anxieties and to overlay French concerns on Canadian issues.

L'influence du nationalisme français au Canada français a fait l'objet de différents travaux avec, en toile de fond, la question, controversée, de la réception et des usages du maurrassisme ${ }^{1}$ ou encore celle des traces du régime de Vichy, marquée par certains dossiers retentissants (affaire Bernonville²). Robert Rumilly (1897-1983), sur lequel une biographie a récemment été publiée ${ }^{3}$, est au cœur de ce dossier. Notre

\footnotetext{
${ }^{1}$ On mentionnera en particulier Catherine Pomeyrols, «Les intellectuels nationalistes québécois et la condamnation de l'Action française ", Vingtième siècle : revue d'histoire, $\mathrm{n}^{\circ} 73$ (janvier-mars 2002), p. 83-98; Pierre Trépanier, «Le maurrassisme au Canada français ", Les Cahiers des Dix, n 53 (1999), p. 167-233; Olivier Dard, "De la rue de Rome au Canada français : influences ou transferts? ", Mens : revue d'histoire intellectuelle de l'Amérique française, vol. VIII, $\mathrm{n}^{\circ} 1$ (automne 2007), p. 7-66; E.-Martin Meunier, "Sur la présumée filiation Groulx / Maurras : contexte politique, enjeu national et écriture de l'histoire ", dans Olivier Dard et Michel Grunewald (dir.), Charles Maurras et l'étranger - L'étranger et Charles Maurras, Berne, PIE Peter Lang, 2009, p. 117-134; Michel Bock, "Linfluence du maurrassisme au Canada français : retour sur le cas de Lionel Groulx ", Ibid., p. 135-152.

${ }^{2}$ Sur ce point, Yves Lavertu, L'affaire Bernonville : le Québec face à Pétain et à la collaboration (1948-1951), Montréal, VLB éditeur, 1994.

${ }^{3}$ Jean-François Nadeau, Robert Rumilly, l'homme de Duplessis, Montréal, Lux éditeur, 2009.
} 
contribution met en lumière les fonctions de passeur et de relais de Robert Rumilly entre le Canada français et la France. Arrivé au Canada en 1928, il en a obtenu la nationalité en 1934 et n'est jamais retourné en France par la suite. Il a fait du Canada sa patrie et s'y est fait un nom, en particulier comme publiciste et comme historien. Pourtant, sur les plans intellectuel et politique, les liens n'ont jamais été rompus avec la France. Durant l'entre-deux-guerres, Rumilly y a publié. Il y a même gagné une petite notoriété si l'on en juge par ses contacts (René Doumic, Henry Bordeaux), le renom de ses éditeurs (Flammarion) et la réception de certaines de ses œuvres. Il y a plus. Son adhésion à l'Action française et notamment à ses troupes de choc, les Camelots du Roi, est revendiquée par Rumilly, qui a souligné l'empreinte de cet engagement et de cette école de pensée sur son itinéraire et ses idées politiques ${ }^{4}$. Jean-François Nadeau a repris cette idée à son compte en considérant que "pour Rumilly, les règles du jeu social, les valeurs, les idées et le projet de société à affirmer sont le corollaire de son idéologie, celle qu'affirme Maurras " . Que Rumilly ait été maurrassien est incontestable, mais une fois la chose dite, les difficultés commencent. Le maurrassisme en effet, s'il possède un noyau doctrinal bien défini, est en même temps divers. Il repose sur des motivations différentes selon les acteurs ou les moments : l'importance propre du facteur politique ne doit pas masquer celle du déterminant religieux, notamment avant 1926 (d'Henri Massis à Jean de Fabrègues), et encore esthétique (Pierre Lasserre). Par ailleurs, le maurrassisme évolue et se reconfigure au gré de la chronologie (Première Guerre mondiale, condamnation de 1926, second conflit mondial), sans compter les crises et les dissidences (Georges Valois, Louis Dimier, Georges Bernanos) qui affectent l'histoire de l'Action française. Toutes ces questions doivent être posées dans le cas de Robert Rumilly.

\footnotetext{
${ }^{4}$ Ibid., p. 62-63.
}

${ }^{5}$ Ibid., p. 64. 
Si le maurrassisme constitue chez Rumilly un ancrage originel, il ne saurait être considéré comme exclusif, en particulier pour comprendre son itinéraire et ses idées après 1945. Dans sa relation à la France, Rumilly se nourrit, en effet, d'autres sources doctrinales que le maurrassisme. Il n'en suit d'ailleurs pas les évolutions, les interrogations et les projets d'aggiornamento portés notamment par Pierre Boutang et sa revue La Nation française (1955-1967). Il se nourrit, en revanche, de la lecture d'autres publications de l'extrême droite hexagonale, en particulier les Ecrits de Paris, principale revue des épurés ${ }^{6}$. Si cette publication compte quelques anciens maurrassiens parmi ses nombreux contributeurs et consacre différents articles à Maurras, elle ne peut néanmoins être considérée comme maurrassienne. Davantage qu'elles ne l'informent sur la France, ces lectures fournissent à Rumilly des grilles interprétatives qu'il transpose et recycle à destination du Canada français. C'est le cas dès l'immédiat après-guerre avec sa brochure de 1949 intitulée La vérité sur la Résistance et l'Épuration en France. Le processus s'opère à une plus grande échelle lorsqu'il se met à publier ses célèbres Cahiers à partir de 1956. S'il mentionne rarement ses sources françaises, une lecture attentive des textes laisse apparaitre le poids de leur influence. Robert Rumilly reprend ainsi à son compte des modes de pensée et des catégories d'analyse hexagonaux pour dénoncer les " gauchistes " canadiens-français, fort de la conviction d'une homologie entre les situations française et canadienne-française. Ainsi, avec un léger décalage chronologique, s'agit-il pour lui de transposer les termes d'une controverse politicoreligieuse qui se joue de l'autre côté de l'Atlantique entre les " catholiques de gauche " et les catholiques conservateurs et traditionalistes. Fort d'un cadre interprétatif, Rumilly possède un atout de poids au début de ses campagnes, mais cet avantage, manifeste à l'époque de sa brochure sur L'infiltration gauchiste au Canada français

\footnotetext{
${ }^{6}$ Les épurés désignent les personnes mises en cause au lendemain du second conflit mondial pour leur attitude vis-à-vis de l'Allemagne durant les années de l'Occupation.
} 
(1956) est de courte durée tant il apparaît que les situations ne sont pas homologues et qu'il aurait fallu, pour rendre son discours davantage crédible, opérer un véritable effort de traduction à destination du Canada français. Familier aux lecteurs français des années 1950 et 1960, le discours sur la subversion, porté sur fond de guerres d'Indochine et d'Algérie dans des milieux aussi bien politiques que militaires ou religieux ${ }^{7}$, l'est sans doute moins aux Canadiens français qui l'ont reçu, même si le maccarthysme a pu les y préparer. Quant aux Français qui auraient pu être friands de ce type de prose, ils n'ont visiblement pas lu ces textes de Rumilly, dont aucune réception de cette partie de son œuvre ne semble attestée.

Trois étapes guideront cet article. Dans un premier temps, il s'agira de dresser un bilan de la relation entre Robert Rumilly, le maurrassisme et le Canada français. Puis, nous nous attacherons à l'action entreprise par Rumilly pour la défense du maréchal Pétain et des collaborateurs, action que nous analyserons à partir de la relation que Rumilly a tentée de nouer avec les défenseurs français des épurés. Enfin, nous étudierons comment Rumilly à partir du milieu des années 1950 s'emploie à transposer et à recycler au Canada français des analyses et des schémas interprétatifs tirés de ses lectures françaises en vue de discréditer le " gauchisme ".

\section{Robert Rumilly, le maurrassisme et le Canada français}

C'est en interrogeant Donatien Frémont (journaliste au Patriote de l'Ouest, où il est entré en 1916, et surtout à La Liberté de Winnipeg depuis $1923^{8}$ ) que Rumilly raconte, dans une sorte de jeu de miroir, son premier contact politique avec le Canada français. Frémont relate ainsi son itinéraire politique en France et son arrivée à Montréal. Après s'être dit marqué par les " persécutions religieuses » du début du $\mathrm{Xx}^{\mathrm{e}}$ siècle en France et avoir rappelé son militantisme à la Ligue

\footnotetext{
${ }^{7}$ François Cochet et Olivier Dard (dir.), Subversion, anti-subversion, contre-subversion, Paris, Riveneuve éditions, 2010.

${ }^{8}$ Robert Rumilly, Chefs de file, Montréal, Les Éditions du Zodiaque, 1934, p. 116.
} 
de la Patrie française et sa lecture, à Nantes, du Nationaliste, il évoque son arrivée sur les bords du Saint-Laurent : «À Montréal, la première chose que je vois dans une vitrine, c'est un journal canadien intitulé Le Nationaliste ${ }^{9}$, et qui adaptait au Canada français des idées très voisines de celles que j'avais servies en France. Alors, j'étais dans une ambiance familière; je me suis tout de suite senti à l'aise. J'attribue à cela ma rapidité d'assimilation ${ }^{10}$. " Le récit de Rumilly reprend la trame de celui de Frémont : " J'ai eu un peu la même expérience. Un peu plus jeune que vous, j'ai adhéré de tout mon cœur non pas à la Patrie française, qui était dissoute depuis plusieurs années, mais à l'Action française. Je lui dois une bonne part, et sans doute la meilleure, de ma formation intellectuelle et morale. Et arrivé à Montréal, je vois sur une boutique de la rue Saint-Denis cette enseigne : l'Action française. Je suis entré, et c'est ainsi que j'ai pris mes premiers contacts avec mes camarades de lettres canadiens ${ }^{11}$. "

L'engagement nationaliste n'est pas le seul point commun revendiqué par les deux hommes. Ils se lancent dans des activités comparables où l'histoire a joué un rôle essentiel. Frémont s'en explique : «En France, j'aurais sans doute fait de la critique : je n'aurais pas été attiré par l'histoire. Mais ici, l'histoire est si riche, si pleine d'enseignements, si utile comme base du patriotisme. Faire de la littérature pure, au Canada, cela se concevrait pour un grand talent. À défaut de cela, il faut avec son petit bout de talent tâcher de servir. "Rumilly fait chorus : "Nous sommes dans le même cas; puisque le même raisonnement m'a fait adopter la même ligne de conduite ${ }^{12}$. "Une " ligne de conduite " qui traduit un constat et un regard porté sur le Canada français : l'histoire y est considérée comme une forme de

\footnotetext{
9 Il s'agit d'un hebdomadaire, organe né en 1904 et qui est l'expression de la Ligue nationaliste lancée en 1903 par de jeunes disciples d'Henri Bourassa (Pascale Ryan, Penser la nation : la Ligue d'Action nationale 1917-1960, Montréal, Leméac éditeur, 2006, p. 26).

${ }^{10}$ Rumilly, Chefs de file, p. 114-115.

${ }^{11}$ Ibid., p. 115-116.

${ }^{12}$ Ibid., p. 120.
} 
ressourcement et le pays comme une France qui n'aurait pas été souillée et où tout serait possible.

$\mathrm{Si}$, comme Frémont, Rumilly appartient au nationalisme français, il est assurément un homme d'Action française. L'empreinte de l'Action française se révèle chez Rumilly à travers de multiples indices qui peuvent s'articuler autour de différents niveaux : politico-littéraire (il parle de Léon Daudet comme " un de [s]es maîtres ${ }^{13}$ " et esthétique (il professe un antiromantisme tout à fait dans la doxa qui, comme Maurras au surplus, ne l'empêche pas d'admirer Lamartine) ${ }^{14}$. Ce maurrassisme, Rumilly l'a revendiqué, et Jean-François Nadeau a utilisé de ce fait les conférences prononcées par Rumilly au Canada français pour en souligner l'importance. Nous versons au débat, pour mieux en cerner les contours, une source essentielle, à savoir une lettre de Robert Rumilly à Maurras, datée du 14 juillet $1937^{15}$ et reproduite ici dans sa quasi-intégralité :

Mon cher Maître,

Je reçois l'Action française du 2, avec votre article sur le Congrès de Québec et sur mon livre sur Marguerite Bourgeoys. [Après les remerciements d'usage, Rumilly enchaîne :]

Quant à votre serviteur, je vous demande la permission après cette introduction (par votre générosité, magistrale) de me présenter un peu, et vous allez voir pourquoi.

Je suis Action française et maurrassien jusqu'à la moelle des os. Lycéen d'Action française à Buffon, avec mon cher ami Marc Dejean de la Bâtie (tué à la guerre), puis à Louis le Grand (prix de philo à ce lycée, Malapert regnante), étudiant d'AF, ligueur $\mathrm{du} \mathrm{XVI}^{\grave{e}}$ avec notre ami Baguenier-Désormeaux. Le trésor de ma bibliothèque est un exemplaire de Romantisme et Révolution ainsi dédicacé : «À monsieur Robert Rumilly, en souvenir des nuits de

${ }^{13}$ Ibid., p. 32.

${ }^{14}$ Nadeau, Robert Rumilly, l'homme de Duplessis, p. 75-78.

${ }^{15}$ Centre d'accueil et de recherche des Archives nationales (CARAN), 576 AP 62, 2 p. dactylographiées. 
garde passées à l'imprimerie d'Action française ". Fils d'officier - et d'une famille toute militaire - mon oncle Vayssière a commandé la garde républicaine -, j'ai fait de belles études prometteuses, j'ai fait la guerre et $j$ 'en suis sorti avec des galons et, ma foi, de belles citations de fantassin (classe 17). Ayant perdu mon père de bonne heure (commandant Rumilly, ancien élève de choix de Foch, mort empoisonné au Tonkin en 1910), j'aurais été tenté par l'anarchie intellectuelle à quoi l'on nous conviait (j'étais boursier de l'État) si je n'avais adopté le maître Maurras et les doctrines - les doctrines $\mathrm{d}^{\prime} \mathrm{AF}$ - auxquels je dois, après mes parents, à peu près tout.

J'ai vécu intensément, et vous savez ce que cela veut dire, toutes les heures de l'Action française. Mais j'en ai eu assez d'aller toujours aux enterrements des nôtres (et le dernier mon camarade Marcel Langlois tué depuis mon départ) et jamais aux enterrements des adversaires. Trop de choses et trop de gens m'ont dégoûté. Malgré vous, malgré vos consignes, j'ai désespéré de jamais voir dans mon pays, dans ma classe sociale, le redressement pour lequel nous avons travaillé. Si j'avais prévu seulement le 6 février, je serais resté. Mais j'ai fini par partir dégoûté de la vieille France pour la nouvelle France.

À quelque chose, malheur est bon. À mon rang, avec mes petits moyens, je me suis livré ici à une œuvre française et je dois vous l'écrire, puisque je le crois, avec plus de confiance dans le succès final que je n'en avais en France même.

C'est moi qui ai conseillé au vaillant TRAVAILLEUR de Worcester d'entrer en relations avec vous. Je suis d'AF. Ne le cache jamais. J'en parle librement chez mes amis les Pères du Saint Esprit, chez mes amis les pères oblats, chez mes amis les pères dominicains. J'ai donné des conférences sur vous à Montréal.

Cinq remarques ressortent à la lecture de ce texte.

La première est que Maurras et Rumilly ne sont pas personnellement liés alors que le « Maître » connaît nombre d'étudiants d'AF (sans parler des vedettes comme Robert Brasillach ou Thierry Maulnier). On rappellera ici que le nom de Robert Rumilly est absent des ouvrages de référence sur l'Action française, comme des principaux 
récits de souvenirs (Dimier, Massis, Valois, etc.) et des Cabiers Charles Maurras qui ont pourtant sollicité durant les années 1960 des témoignages d'étrangers.

Le courrier de Rumilly illustre l'ancienneté de son engagement - lycéen d'AF (catégorie choyée par Maurras avant 1914) - et balaie les digressions de Jean-François Nadeau sur les supposés tâtonnements de Rumilly qui, selon lui, «n'est pas conquis d'emblée par l'Action française ${ }^{16}$ " et aurait même eu une tentation anarchiste. Parlons net : Rumilly n'a pas eu l'itinéraire de Valois et de ses disciples et n'a pas fréquenté les milieux du cercle Proudhon.

On relève encore que Rumilly est un militant engagé mais un militant de base de l'AF, qui participe de ce fait à une des tâches classiques : la garde de l'imprimerie. Il a participé à nombre d'actions militantes, mais son seul fait d'éclat connu et célébré par lui est la mise sur pied d'un chahut à l'occasion de l'élection manquée de Maurras à l'Académie française contre Célestin Jonnart en $1923^{17}$. Rumilly l'a largement raconté et a même fait encadrer dans son salon la caricature de Sennep parue le lendemain dans l'Action française. Le fait est authentique, mais sa portée doit être relativisée : l'année 1923 est dominée à l'AF par l'assassinat de Marius Plateau (secrétaire général des Camelots du Roi) et la mort controversée de Philippe Daudet. De fait, le coup d'éclat de Rumilly est absent des travaux sur Maurras (y compris de la biographie de Stéphane Giocanti) et surtout de l'Almanach d'Action française de 1924, qui s'étend en revanche largement sur l'assassinat de Plateau et donne à voir, photos à l'appui, le saccage de l'Ere nouvelle et de l'CEuvre. Si l'on cherche des occurrences sur Rumilly dans l'orbite maurrassienne, elles sont bien rares. On notera que Rumilly a publié en 1932, dans la Revue universelle et sous la rubrique "La vie à l'étranger ", une chronique sur " Les survivances françaises en Amérique du Nord ${ }^{18}$ ». Informatif,

\footnotetext{
${ }^{16}$ Nadeau, Robert Rumilly, l'homme de Duplessis, p. 58.

${ }^{17}$ Stéphane Giocanti, Maurras : le chaos et l'ordre, Paris, Flammarion, 2006, p. 285.

${ }^{18}$ Revue universelle, 15 décembre 1932, p. 734-741.
} 
ce texte ne donne guère à penser que Rumilly est un ancien de la rue de Rome. On ajoutera même que Rumilly est moins favorisé dans la revue de Jacques Bainville que dans la Revue des deux mondes de René Doumic, qui lui ouvre ses colonnes pour un article complet sur sir Wilfrid Laurier ${ }^{19}$. Si l'on ajoute que son ouvrage sur Papineau est publié chez Flammarion en 1934, on peut considérer que, vu de France au milieu des années 1930, beaucoup plus qu'un maurrassien français installé outre-Atlantique qui proposerait à ses homologues français une analyse idéologiquement typée du Canada, Rumilly incarne par ses écrits une figure de publiciste dont les essais biographiques permettent à ses lecteurs hexagonaux de se familiariser avec l'histoire du Canada français.

En quatrième lieu, soulignons une faute qui échappe à Rumilly mais qui donne la mesure, malgré la revendication de son maurrassisme, de son éloignement des milieux de l'Action française. Il explique ainsi à Maurras les raisons de son retrait : les morts non vengés... et revient sur le 6 février. En fait, sans vouloir sans doute blesser son " Maître" mais en ne mesurant pas bien la portée de son propos pour le destinataire, Rumilly reprend à son compte le procès fait par des dissidents de l'AF (d'Henri Martin ${ }^{20}$ aux futures imprécations de Lucien Rebatet dans les Décombres sur son « inaction $»^{21}$ ), des années 1920 au 6 février 1934, malgré la violence verbale et écrite. Maurras, Daudet ou

19 "Un "premier" de race française, au Canada : Sir Wilfrid Laurier ", Revue des deux mondes, $1^{\text {er }}$ octobre 1931 , p. 570-591.

${ }^{20}$ Il s'agit du docteur Martin que l'on retrouve, quelques années plus tard, dans la Cagoule.

${ }^{21}$ Lucien Rebatet, critique de cinéma en vue et journaliste politique engagé, passé du maurrassisme au fascisme et à l'ultra collaboration à l'instar de nombre de ses amis de l'hebdomadaire Je suis partout, a réglé ses comptes avec l'Action française dans son pamphlet autobiographique Les décombres paru en 1942 chez Denoël et qui fut le succès éditorial majeur de l'Occupation. Dans un chapitre intitulé " $\mathrm{Au}$ sein de l'inaction française ", Rebatet fustige durement l'impuissance politique de l'Action française qui, selon lui, aurait stérilisé bien des énergies militantes. Rappelant l'assassinat de Marius Plateau, il relève : "Elle effrayait la République. Mais elle avait commencé à la rassurer en laissant tuer ses hommes sans riposter " (p. 112-113). 
Maurice Pujo (le patron des Camelots du Roi) ont déjà répondu longuement sur ce point et raillé, à l'instar de ce dernier, « certains royalistes " qui « voudraient $[. .$.$] changer de route » et voient l'Action$ française comme un "canapé de doctrinaires ${ }^{22}$ ".

En cinquième lieu, le texte donne la mesure de la place du Canada français dans la vision de Rumilly : mener un combat similaire sous d'autres cieux... en considérant que l'Amérique française est une France idéalisée où tout serait possible car rien n'aurait été détruit : pour parler en langage maurrassien, un "pays réel " pas trop abîmé par le "pays légal ". Ainsi, Robert Rumilly a-t-il décrit sir Wilfrid Laurier comme «le type d'homme le plus parfait produit par la race française en Amérique ${ }^{23}$ ". Cette Amérique, Rumilly l'a arpentée de Boston à Saint-Boniface. Il consacre d'ailleurs à une figure francoaméricaine importante, Elphège Daignault, l'un des portraits de ses " chefs de file ». L'homme est né aux États-Unis et est une personnalité majeure de la ville de Woonsocket (elle compte alors 50000 habitants) surnommée la "Québec de la Nouvelle-Angleterre ». Rumilly présente complaisamment sa démarche : "Nous sommes aux États-Unis les continuateurs et les propagateurs de l'idéal catholique et français. Et nous croyons enrichir ainsi la vie nationale, la civilisation américaine en voie de formation ${ }^{24}$. " Rumilly tient surtout à témoigner et à se ressourcer: « Nous avons vu, de nos yeux vu, des réunions de francoaméricains $[s i c]$ où l'entrain, l'enthousiasme, l'atmosphère française passaient tout ce qu'on imagine. Au banquet du Travailleur, par exemple, nous nous sommes cru transporté dans une des vibrantes réunions parisiennes de l'Action française. Ici comme là, une pléiade intrépide se trouvait réunie autour d'un chef jouissant de la confiance unanime."

En résumé, l'Action française a donné à Rumilly un bagage de références, une vision de la France et du monde. Elle lui fournit aussi

\footnotetext{
${ }^{22}$ Maurice Pujo, "Le chemin de la victoire ", discours prononcé au banquet des étudiants d'Action française, Almanach de l'Action française, 1936, p. 134.

23 "Un "premier" de race française, au Canada : Sir Wilfrid Laurier ", p. 591.

${ }^{24}$ Rumilly, Chefs de file, p. 65.
} 
un modèle en matière de propagande dont il tente de convaincre l'abbé Groulx en soulignant, auprès de lui, la nécessité de lancer une forme de Revue universelle à la canadienne et de ne pas s'en tenir à la seule Action nationale. Rumilly lui en souligne les limites et lui propose, sans le convaincre, une solution directement inspirée de l'exemple maurrassien :

À ce sujet [l'Action nationale], j'aurais pour ma part souhaité une revue qui tout en cherchant à répandre une doctrine n'en eût pas fait l'objet unique de ses articles. Quelque chose comme la Revue universelle de Jacques Bainville et d'Henri Massis. On n'y perd pas une occasion de prêcher la bonne parole, mais les lecteurs y trouvent aussi une grande variété d'articles : littérature, reportages, etc. On attire ainsi un plus grand public, des gens qui cherchent dans la lecture une détente. Et l'on fait de nouvelles recrues; on fait acte de propagande. Alors qu'un organe purement patriotique ne touche que les partisans, les convaincus. Ainsi, l'on manque de communication avec le peuple ${ }^{25}$.

Maurrassien déclaré, ambassadeur autoproclamé de l'Action française, Rumilly agit comme un électron libre. Il n'est mandaté par personne, et son importance dans l'histoire du maurrassisme doit être comprise à cette aune. Le maurrassisme de Rumilly est une construction personnelle et datée qui éclaire la première partie de son itinéraire. Par la suite, tout en restant dans le giron de ce qu'il est convenu d'appeler l'extrême droite, Rumilly renouvelle ses références et se coupe largement du maurrassisme et des maurrassiens après le second conflit mondial.

\section{Rumilly, la France et l'épuration}

Durant la guerre, Robert Rumilly est un pétainiste convaincu et le demeure par la suite en apportant son soutien au maréchal Pétain et à ses collaborateurs. Dès le début de 1945, il envisage de faire signer

${ }^{25}$ Ibid., p. 127. Si la Revue universelle a un tirage important (8 000 ex.), elle ne touche pas le "peuple ". Quant au fait qu'on ne "perd pas une occasion de prêcher la bonne parole ", les choses sont un peu plus compliquées. 
et diffuser une protestation et s'en ouvre à Camillien Houde, qui hésite à lui donner sa signature pour son projet dénonçant "les exécutions, procès et condamnations que subit présentement une partie de l'élite française ${ }^{26}$ ". Les opinions de Rumilly sur l'épuration sont publiées à la une de journaux, comme en atteste cette "lettre " parue dans Vers demain où il félicite son directeur pour un article intitulé "Pétain, martyr de la patrie ». Le diagnostic de Rumilly est sans appel : "De la juiverie à la maçonnerie en passant par l'Intelligence Service, toutes les forces qui s'acharnent contre Pétain, comme elles s'acharnent contre Franco, sont ennemies mortelles des traditions et des aspirations du Canada français. Ceux des nôtres qui y prêtent les mains sont des fous ou des traîtres ${ }^{27}$. " Le maréchal Pétain n'est pas le seul objet de préoccupation de Rumilly, qui se soucie également du sort des collaborateurs réfugiés au Canada français.

Il n'est pas question de reprendre ici l'ensemble de cette affaire mais plutôt de montrer, en nous appuyant sur les archives de Rumilly, comment ce dernier articule son rapport à l'épuration, qui le replonge au cœur de polémiques franco-françaises ${ }^{28}$. L'ambiguïté de sa situation ne lui échappe pas et il s'en explique dans une conférence publiée en brochure. Dès ses premiers mots, Rumilly prend les devants et souligne: "Je n'ai pas l'habitude et je n'ai pas l'intention de transporter ici des querelles françaises. Mais il s'est passé, il se passe encore des choses d'une épouvantable gravité que les propagandes et les censures officielles taisent, cachent et quelquefois nient [...]. » C'est pour briser ce mur du silence que Rumilly prend la parole, fort de se considérer " en communion profonde avec l'immense majorité des

${ }^{26}$ Lettre du 20 février 1945, Bibliothèque et Archives nationales du Québec - Centre de Montréal (à l'avenir BAnQ-M), Fonds Robert Rumilly, D 1/9.

${ }^{27}$ Vers demain, 15 août 1945 , p. 1.

${ }^{28}$ Sur ces points, voir en particulier Peter Novick, L'épuration française, 1944-1949, Paris, Les Éditions Balland, 1985; Jean-Paul Cointet, Expier Vichy: l'épuration en France (1943-1958), Éditions Perrin, 2008; Bénédicte Vergez-Chaignon, Vichy en prison : les épurés à Fresnes à la libération, Paris, Gallimard, 2006, et Histoire de l'épuration, Paris, Larousse, 2010. 
Canadiens français, mes compatriotes, mes frères ${ }^{29}$ ". La prose de Rumilly renvoie à une vision horrifiante de la résistance et en particulier des maquis tout comme de l'épuration. À dire vrai, elle n'est nullement originale eu égard à ce qui se publie alors en France du côté des épurés. On y retrouve en particulier des descriptions d'exécutions et de règlements de comptes, de procès bâclés, sans compter les chiffres fantaisistes des exécutions sommaires: pas plus que le parti communiste n'a compté 75000 fusillés, il n'y.a eu en France entre 80000 et 100000 exécutions sommaires. Ce qui est notable chez Rumilly, c'est la thèse générale qu'il propose. De même qu'il considère que la résistance a été l'affaire des communistes (il semble ignorer son pluralisme et même le fait que des maurrassiens - Daniel Cordier, Guillain de Bénouville, Rémy, etc. - y ont largement participé), il estime que l'épuration est " une vaste opération politique conduite par le parti communiste avec la complicité au moins tacite de pieux ministres comme Francisque Gay pour liquider les éléments de droite ${ }^{30}$ " (ce qui revient à ignorer la part des gauches dans la collaboration, en particulier sous l'angle du collaborationnisme). On ne négligera nullement la dimension idéologique de la démarche de Rumilly. Son souci propagandiste est évident et il s'emploie à mettre en scène des affaires particulièrement sordides. Il entend frapper ses interlocuteurs et imprimer dans leur esprit des continuités entre, notamment, la répression anti-ecclésiastique conduite par les républicains espagnols et l'épuration, ce qui lui a valu d'être avantageusement cité dans une chronique diffusée le 22 février 1949 par la Radio nationale d'Espagne dans ses émissions internationales ${ }^{31}$. Il y a aussi beaucoup de méconnaissance chez Rumilly. Ce dernier travaille en effet de seconde main à partir de quelques organes de presse engagés

${ }^{29}$ Robert Rumilly, La vérité sur la Résistance et l'Épuration en France, 1949, p. 1. Il n'y a ni lieu de publication ni éditeur. La seule mention figurant à la fin de la brochure renvoie à l'adresse de Robert Rumilly, laissant à penser qu'il s'agit d'une brochure publiée à compte d'auteur.

${ }^{30}$ Ibid., p. 10.

${ }^{31}$ Pierre Desjardins, "L'intelligence canadienne ", lettre du 24 septembre 1948, BAnQ-M, Fonds Robert Rumilly, D 1/11. 
contre l'épuration, notamment Paroles françaises de l'ancien résistant André Mutter, La France catholique de Jean de Fabrègues ou des témoignages publiés, dont celui bien connu du chanoine Desgranges ${ }^{32}$. Conscient de ses limites, Rumilly souhaite aller plus loin et tente de nouer le contact avec les principales figures françaises de ce combat. La correspondance est ici instructive et montre qu'il a cherché à monter tout un réseau de défense franco-canadien qui serait passé par l'échange d'information, la diffusion de publications diverses et, surtout, la mise sur pied de conférences.

On retiendra en particulier les contacts ébauchés avec Paroles françaises au sujet de l'affaire Bernonville après que Jean-Marie SaintMéran, son rédacteur en chef, eut pris attache avec Camillien Houde. La réponse du maire de Montréal est une excellente introduction pour Rumilly qu'il charge de " fournir les détails demandés " en ajoutant " affirmer que tout ce que Monsieur Rumilly vous fournira, sera d'une stricte exactitude. Je fais miennes les opinions qu'il pourra vous exprimer, au sujet de M. de Bernonville ${ }^{33}$ ». Rumilly s'y emploie et cherche, en échange de renseignements, à en obtenir d'autres. Si Paroles françaises a publié un article, cité Rumilly et, surtout, par la suite fait état de ses conférences ${ }^{34}$, la réponse du secrétariat de la rédaction de Paroles françaises est décevante : « Malgré notre désir de vous être agréable, nous ne pouvons vous fournir les coupures de journaux communistes que vous nous demandez, ces derniers ayant d'ailleurs fort peu parlé de l'affaire ${ }^{35}$. " Dans d'autres cas, Rumilly a davantage de succès, et le député conservateur Joseph Denais lui a adressé une note de renseignements sur la carrière de Francisque Gay que Rumilly a reprise dans sa conférence précitée $e^{36}$. Ajoutons encore

\footnotetext{
${ }^{32}$ La brochure précitée de Rumilly est une compilation de coupures de presse que l'on retrouve dans ses archives.

${ }^{33}$ Lettre du 16 septembre 1948, BAnQ-M, Fonds Robert Rumilly, D 1/10.

34 "L'affaire Bernonville", Paroles françaises, 17 septembre 1948. Voir aussi "Francisque Gay doit quitter le Canada! ", Paroles françaises, 5 décembre 1948, consacrant un long passage à la conférence de Rumilly, qui est aussi longuement cité.

${ }^{35}$ Lettre du 24 septembre 1948, BAnQ-M, Fonds Robert Rumilly, D 1/10.

${ }^{36}$ Lettre du 31 octobre 1948, BAnQ-M, Fonds Robert Rumilly, D 1/10.
} 
que L'Indépendance française lui envoie quelques renseignements chiffrés ${ }^{37}$ et a rendu compte de La vérité sur la Résistance et l'Épuration en France $e^{38}$.

Le bilan, à lire Rumilly lui-même, est en demi-teinte. Remerciant Joseph Denais, il lui explique que « contrairement à [s] on habitude d'historien ", il est " réduit à pinocher [s] a documentation à droite et à gauche parmi les textes déjà publiés ${ }^{39}$ ". Rumilly s'ouvre davantage au jeune directeur d'Aspects de la France et du Monde, Pierre Boutang. On y retiendra d'abord ces propos de l'ancien « ligueur " sur la tentative de Boutang de redonner du lustre au maurrassisme : "J'admire à la fois le courage dont vous faites preuve en relevant le drapeau de l'Action française (vous avez l'espoir chevillé à l'âme!) et le résultat obtenu. Aspects, dans sa nouvelle formule, n'est pas indigne de l'Action française. Peut-être un peu uniformément sévère, de toilette et de ton. "L'essentiel de sa lettre n'est pas là, mais dans sa volonté d'obtenir de l'information. Il avoue être sur ce point "assez sevré ", ajoutant : «Et les intéressés, de France, ne nous aident pas dans ce travail accompli pour eux. Je me suis adressé à plusieurs endroits sans grand succès. Paroles françaises répond peu à mes demandes. Il faudrait, d'une manière générale, nous envoyer des renseignements - des listes de faits sûrs - établissant les atrocités de la Libération, les iniquités de la "Justice", le caractère communiste de toutes ces saloperies ${ }^{40}$. " Suit une liste de questions précises auxquelles on ne sait si Boutang y a répondu (on peut en douter). Ce constat dressé par Rumilly vaut

\footnotetext{
${ }^{37}$ Lettre de la direction de l'Indépendance française à Rumilly, 12 novembre 1949, qui reprend le chiffre de 80000 fusillés (BAnQ-M, Fonds Robert Rumilly, P 303 S2 SS 11).

${ }^{38}$ Robert Rumilly reçoit à ce sujet un courrier de J. Gravereau, maire adjoint de l'Hayles-Roses, qui se propose de lui acheter cette brochure après en avoir lu " un compte rendu élogieux " dans l'Indépendance française paru la veille (BAnQ-M, Fonds Robert Rumilly, P 303 S2 SS 11). On sait également qu'une librairie parisienne à laquelle il avait envoyé 6 brochures lui en a commandé 12 par un courrier du 6 mars 1950, tandis que l'Union des industriels du Loiret lui en commande 100 (BAnQ-M, Fonds Robert Rumilly, P 303 S2 SS 11).

${ }^{39}$ Lettre du 17 novembre 1948, BAnQ-M, Fonds Robert Rumilly, P 303 S2 SS 11.

${ }^{40}$ Lettre non datée, BAnQ-M, Fonds Robert Rumilly, D 1/9.
} 
aussi pour les tentatives auxquelles il a été associé dans le but de faire venir des conférenciers de renom au Canada français. À l'été 1949, les noms de Jacques Isorni (l'avocat de Brasillach et de Pétain) comme celui du chanoine Desgranges sont évoqués dans des échanges où sont impliqués René Malliavin (fondateur des Écrits de Paris), Louis Rougier (envoyé par Pétain en mission à Londres, antigaulliste farouche) et Robert Giacometti : la chose est jugée "impossible " pour le premier, "car il est divorcé ", tandis que le second est "fatigué " ${ }^{41}$. L'année suivante, un contact noué directement entre Rumilly et le colonel Rémy n'a pas donné les résultats attendus ${ }^{42}$. Nous n'en connaissons pas les raisons, mais on peut se demander si certains courriers adressés à Rémy par des proches de Rumilly ne l'ont pas indisposé, comme celui envoyé par Philippe Hamel et qui fixait à Rémy une feuille de route peu compatible avec sa démarche de réconciliation entre Londoniens et Vichyssois ${ }^{43}$ : "Si vous deviez venir au Canada pour faire l'apologie de Pétain et ignorer de Gaulle, ou mieux encore, le dénoncer, vous rendriez un service immense à la vérité et vous seriez accueilli avec enthousiasme ${ }^{44}$. "

\section{Rumilly, des " catholiques de gauche " à la subversion : placage et réinterprétation}

De la publication de L'infiltration gauchiste au Canada français à Quel monde!, Rumilly inaugure une nouvelle phase de son itinéraire politico-intellectuel. Sans que le mot soit à l'origine directement prononcé, il est le pourfendeur de la subversion, une subversion de grande envergure qui attaquerait et démantèlerait une société pourtant saine, celle du Canada français. Pendant dix ans, à travers

${ }^{41}$ Lettre du 13 août 1949 de Christian Wolf à Robert Giacometti, qui la transmet à Rumilly, BAnQ-M, Fonds Robert Rumilly, D 1/12.

${ }^{42}$ Lettre du 21 août 1950 du colonel Rémy à Rumilly, BAnQ-M, Fonds Robert Rumilly, D $1 / 13$.

${ }^{43}$ Voir en particulier Henry Rousso, Le syndrome de Vichy, 1944-198..., Paris, Seuil, 1987, et Bénédicte Vergez-Chaignon, Les vichysto-résistants de 1940 à nos jours, Paris, Éditions Perrin, 2008.

${ }^{44}$ Lettre du 31 octobre 1948, BAnQ-M, Fonds Robert Rumilly, P 303 S6 SS, D 1/13. 
une série de brochures, de conférences et d'interviews, il ressasse le thème. L'audience de ses propos est importante (10 000 exemplaires diffusés pour L'infiltration gauchiste au Canada français $\left.{ }^{45}\right)$ même si elle a tendance à décliner au début des années 1960 (Quel monde! est imprimé à 4050 exemplaires sur lesquels 1880 ont été invendus et retournés $)^{46}$.

Ces différentes brochures de Rumilly sont construites sur le même modèle, à savoir une collection de citations tirées de la presse et visant à établir les méfaits du " gauchisme » au Canada français. Des quotidiens, à commencer par Le Devoir, Radio-Canada, des figures renommées, tant laïques qu'ecclésiastiques, sont particulièrement visés. C'est moins aux cibles désignées qu'à l'argumentation déployée et à ses origines que nous nous attacherons. La thèse de Rumilly est simple, martelée dans chacun de ses opuscules et souvent, à différentes reprises, au sein du même ouvrage : le "gauchisme " serait une importation française émanant des " catholiques de gauche " et tout particulièrement de la revue Esprit, dont Cité libre serait la " petite sœur ». Le lien pointé par Rumilly est réel, et l'historiographie récente l'a largement souligné ${ }^{47}$. Ce qu'il faut cependant mettre en lumière, c'est le mode de fabrication déployé. La lecture croisée de la prose de Rumilly et de certains des auteurs et des publications majeures de l'extrême droite française des années 1950 et 1960 montre comment s'opère, par l'intermédiaire de Rumilly, à la fois une transposition et un recyclage. Si les cité-libristes puisent une bonne part de leur inspiration dans Esprit, au milieu des années 1950 Rumilly trouve la sienne chez Jean Madiran et dans les Écrits de Paris.

${ }^{45}$ Sur la diffusion et la réception de ce dernier au Canada français, voir Xavier Gélinas, La droite intellectuelle québécoise et la Révolution tranquille, Québec, Les Presses de l'Université Laval, 2007, p. 91-94.

${ }^{46}$ Les chiffres sont ceux du 9 juin 1966 et accompagnent une lettre à Rumilly du 9 juillet 1966 que lui a adressée Jean-Guy Genest, le directeur des éditions Actualité (BAnQ-M, Fonds Robert Rumilly, P 303 5J SS13).

${ }^{47}$ Stéphanie Angers et Gérard Fabre, Échanges intellectuels entre la France et le Québec (1930-2000): les réseaux de la revue Esprit avec La Relève, Cité libre, Parti pris et Possibles, Québec, Les Presses de l’Université Laval, 2004. 
Jean Madiran (Jean Arfel à l'état civil) est né en 1920. Il est connu aujourd'hui comme le fondateur de la revue Itinéraires et un des piliers du quotidien catholique traditionaliste Présent, qu'il a contribué à lancer en 1982. Jean Madiran vient de l'Action française (il a été le secrétaire particulier de Maurras) et a écrit, sous le pseudonyme de Jean-Louis Lagor, dans la presse monarchiste d'après-guerre (L'Indépendance française). Rumilly connaît ce journal et lit surtout les Écrits de Paris, revue lancée en 1947 pour défendre en particulier les épurés et qui ne saurait être considérée comme une revue catholique même si elle a publié des textes de Jean Madiran que Rumilly a réutilisés ${ }^{48}$.

Les sources de Robert Rumilly éclairent son horizon intellectuel, ses méthodes de travail et le contenu même de son argumentation. Lire Robert Rumilly permet à l'historien des idées de croiser des figures connues et répertoriées. Les références de Rumilly évoluent d'ailleurs avec le temps, montrant par là qu'il creuse moins un sillon qu'il ne cherche des points d'ancrage pour affermir sa démonstration, transposer et recycler schémas et références venus de France. L'objectif est clair : proposer aux Canadiens français une grille d'interprétation sur les menaces qui pèsent sur eux puisqui il serait établi que le Canada français est le futur de la France et que ce qui s'observe dans l'Hexagone est appelé à se produire au Canada français. Dans l'Infiltration gauchiste au Canada français, Rumilly s'explique et se justifie : "Le présent travail, destiné à l'examen d'une situation canadienne, ne tente, en prologue, qu'une incursion indispensable dans la vie politique ou intellectuelle de la France ${ }^{49}$."

${ }^{48}$ Selon Rumilly, qui la lit depuis l'origine, la revue ne compte que quelques abonnés au Canada français (Robert Rumilly, Mon cahier $n^{\circ} 1$ : l'infiltration gauchiste au Canada français, édité par l'auteur, 1956, p. 134). Il évoque aussi "d'excellentes revues catholiques " en France et mentionne les Écrits de Paris et La France catholique (Ibid., p. 68-69). Si la seconde est incontestablement une revue catholique, cette dénomination ne saurait s'appliquer à la première.

${ }^{49}$ Rumilly, Mon cabier $n^{\circ} 1$ : l'infiltration gauchiste au Canada français, p. 22. 
Le premier combat que conduit Rumilly porte sur Esprit et son relais canadien-français Cité libre. Fort significativement, Rumilly ne part pas de la situation canadienne-française mais de la situation hexagonale. Le lecteur canadien y est donc transporté au milieu d'une controverse qui oppose notamment Jean Madiran aux dirigeants d'Esprit, considérés comme des crypto-communistes. La prose antiprogressiste de Rumilly décalquie celle de Madiran et n'en a d'ailleurs pas la densité. Si on veut y trouver un élément d'originalité, il réside dans l'emploi du terme "gauchiste ", inusité en France à l'époque, et dans les soucis de Rumilly de revenir encore et toujours sur la défense du maréchal Pétain et la dénonciation de l'épuration. Religion et politique sont chez Rumilly intimement liées, tant pour la France que pour le Canada français : " Nous avons vu que les "catholiques de gauche", en France, ont adopté le thème de la lutte des classes, article numéro 1 du bréviaire marxiste. Et nous verrons plus loin que Le Devoir s'inspire auprès des "catholiques de gauche". Le Devoir adopte lui aussi le thème de la lutte des classes ${ }^{50}$. "Plus encore, Le Devoir est considéré comme le simple décalque d' $E s p r i t^{51}$. En même temps qu'il est fondé sur un raisonnement par analogie, le propos de Rumilly se veut une mise en garde. L'objectif est d'allumer un contrefeu en refusant que les adversaires des " catholiques de gauche " soient ignorés (Henri Massis) ou déconsidérés avant même d'avoir été discutés (Jean Madiran) : «Les citations du Devoir sont terriblement partiales. Sous le titre "Ils ne savent pas ce qu'ils font", Jean Madiran a publié un livre retentissant ${ }^{52}$, pour exposer et combattre les manœuvres des "catholiques de gauche", qui préparent l'infiltration communiste dans les milieux catholiques de France. Le Devoir se garde bien d'en parler. Mais il reproduit l'article de Témoignage chrétien qui, à vrai dire, ne cherche même pas à réfuter Madiran mais tente de le tourner en ridicule : "rigolade... Il y a de quoi voler en éclats de

\footnotetext{
${ }^{50}$ Ibid., p. 50.

${ }^{51}$ Ibid., p. 67-70.

${ }^{52}$ Rédigé entre août et décembre 1954, l'essai de Jean Madiran Ils ne savent pas ce qu'ils font a été publié aux Nouvelles Éditions latines en 1955.
} 
rire...", etc. Une petite note de la rédaction du Devoir endosse l'article de Témoignage chrétien : elle tend à indiquer ce qui est valable non seulement pour la France, mais pour le Canada ${ }^{53}$. »Comme à son habitude, Robert Rumilly se met en scène :

Que le lecteur me permette une réflexion un peu personnelle. La lutte des classes a empoisonné l'atmosphère française. Elle a divisé les Français, les a dressé[s] les uns contre les autres; elle a aigri les rapports quotidiens au point de créer un état larvé de guerre civile. C'est la principale raison qui m'a fait quitter la France pour le Canada, il y a près de trente ans. J'ai trouvé ici, comme je le prévoyais, une France que la lutte des classes n'avait pas gâtée. Je puis prendre un taxi, m’asseoir à côté du chauffeur et bavarder pendant la course. Je puis, sur un chantier, partager le casse-croûte d'un briqueteur ou d'un menuisier. Ces rapports sont à peu près inconcevables en France, où mon seul physique d'intellectuel à lunettes ferait repérer en moi un "sale bourgeois ", un ennemi. Le chauffeur et moi, le briqueteur ou le menuisier et moi serions aussi gênés l'un que l'autre; nous ne parlerions pas la même langue. Eh bien, la situation est en train d'évoluer au Canada français. Le Devoir et les dirigeants des syndicats catholiques l'affaire est trop grave pour que je n'écrive pas toute ma pensée, pour que je n'avertisse pas tous les responsables - sont en train de créer, délibérément, une mentalité de classes, d'acclimater la haine des classes. Je viens de vous donner une idée du résultat qu'ils obtiendront ${ }^{54}$.

Plus largement, le constat que fait Rumilly est celui d'une dénaturation qui concerne non seulement le catholicisme mais également le nationalisme : "Les catholiques de gauche, en France, sont devenus plus gauchistes que catholiques. Les "nationalistes de gauche", au Canada, deviendront plus gauchistes que nationalistes ${ }^{55}$."

\footnotetext{
${ }^{53}$ Rumilly, Mon cahier $n^{\circ} 1$ : l'infiltration gauchiste au Canada français, p. 70. On notera que Madiran déplore lui aussi une conspiration du silence.

${ }^{54}$ Ibid., p. 51-52.

${ }^{55}$ Ibid., p. 122.
} 
Les Cahiers suivants de Robert Rumilly s'appuient sur les mêmes fondements et reprennent les mêmes thématiques en les actualisant. On retrouve ainsi l'accent mis sur la France : "Ce sont les gauchistes qui ont importé de l'étranger, principalement de France, les idées et les méthodes avec lesquelles ils ont introduit la division profonde qui déchire aujourd'hui la pensée canadienne-français $e^{56}$. " Les " catholiques de gauche " sont toujours aussi nettement dénoncés. Ainsi, Rumilly fustige-t-il l'abbé Pierre pour « avoir voté contre un projet d'amnistie aux fidèles du Maréchal Pétain » et dénoncé les « soi-disant tortures infligées par l'armée française à des prisonniers en Algérie " en même temps qu' il ne disait mot du sort des catholiques au Tonkin ou des victimes de Budapest. Le mouvement porté par l'abbé Pierre et qui serait d'ampleur internationale (Rumilly évoque aussi bien le Portugal que l'Amérique du Sud) est celui d'un vaste " réseau » au service d'une entreprise de "propagande subversive ${ }^{57}$ ». On ajoutera enfin les nombreuses prises de position sur la vie politique française, qui voient Rumilly se situer dans une opposition permanente, du gouvernement Mendès France à Charles de Gaulle. Concernant Mendès, on retrouve dans le propos de Rumilly une analyse tout à fait française et politiquement marquée lorsqu'il utilise l' " affaire Didès » (sic) pour accuser Mendès de philo-communisme ${ }^{58}$ et ironiser sur « '"“espoir" » représenté par Mendès selon Le Devoir : «À qui Le Devoir fera-t-il croire que le sort de la France est lié à celui du Juif franc-maçon Mendès, entouré d'une équipe de Juifs maçons et communisants $^{59}$ ? " Pour ce qui est de Charles de Gaulle, les griefs accumulés depuis le second conflit mondial et la politique algérienne du général scellent un antigaullisme profond.

\footnotetext{
${ }^{56}$ Robert Rumilly, Mon cabier $n^{\circ} 3$ : les socialistes dominent le réseau gauchiste, édité par l'auteur, 1959, p. 158.

${ }^{57}$ Le mot " réseau " fait même l'objet d'un chapitre (Ibid., p. 129 et suiv.), et l'expression " propagande subversive " est citée p. 156.

${ }^{58}$ Cette affaire a marqué des soutiens de Mendès comme Bertrand de Jouvenel (Olivier Dard, Bertrand de Jouvenel, Paris, Éditions Perrin, 2008, p. 305-306).

${ }^{59}$ Rumilly, Mon cabier $n^{\circ} 1$ : l'infiltration gauchiste au Canada français, p. 73.
} 
L'impression de répétition offerte par la prose de Robert Rumilly doit cependant être corrigée à la lecture attentive de ses écrits. On constate en effet, en examinant en détail le contenu de Quel monde! paru en 1965, une inflexion qui conduit Rumilly du côté de la revue française Europe-Action dirigée alors par Dominique Venner et axée sur la défense de la "race blanche " ou l'opposition de "l'homme blanc " et des "peuples de couleur ${ }^{60}$ ". Certes, aucun auteur ni aucun texte ne sont cités, mais on trouve sous la plume de Rumilly des formules qui sont des reprises quasi identiques de titres d'articles ou de dossiers parus dans Europe-Action. Par exemple, "la chasse à l'homme blanc est commencée dans certaines parties de l'Afrique" renvoie sans nul doute au dossier intitulé "La chasse aux blancs" publié en janvier 1965 dans le ${ }^{\circ} 25$ d'Europe-Action ${ }^{61}$, de même que les développements consacrés à Malcom X. L'influence d'EuropeAction se mesure aussi à travers des références positives à Saint-Loup et à ses ouvrages (Les Volontaires...). Plus piquants encore sont les développements que consacre Rumilly à l'opposition entre l'Occident et les barbares en se référant à Guillaume II auquel il espère que la " postérité rendra peut-être à [sa] mémoire [...] la dose de justice qui lui est due ${ }^{62}$ ". L'ancien maurrassien aurait-il oublié ses classiques? En fait, ces notations invitent à remettre en perspective la place revêtue par la doctrine chez lui. Elle n'est ni exclusive ni essentielle, et la démarche de Rumilly doit être comprise comme celle d'un syncrétisme. C'est une constante chez lui qu'il revendique de façon explicite. Ainsi, commentant les divergences entre l'Alliance laurentienne et le Rassemblement pour l'Indépendance nationale de même que les débats sur le "souverainisme " et le "séparatisme ", relève-t-il : "Tout en m'efforçant de traduire ma pensée dans les mots d'une manière aussi précise que possible, je n'attache pas une importance démesurée aux

${ }^{60}$ Robert Rumilly, Quel monde! Communisme! Socialisme! Séparatisme!, Montréal, Les éditions Actualité, 1965, p. 3-5.

${ }^{61}$ Ibid., p. 7-8.

${ }^{62}$ Ibid., p. 9-10. 
querelles de vocabulaire qui m'ont paru toujours un peu byzantines ${ }^{63}$. " Dans ces conditions, les oppositions repérables au sein de l'extrême droite française entre notamment Europe-Action et Jeune Révolution sur le sort de l'Afrique, ou les régimes ibériques s'effacent chez Rumilly. Son objet est de mobiliser voire de fédérer des oppositions autour d'un ennemi commun incarné dorénavant par la "subversion ", que Rumilly décline sous ses différents aspects (politique, musical ou religieux $^{64}$ ) et ses différents théâtres, du Congo au Québec. À le lire, "le peuple canadien-français ", "peuple le plus sain du monde ${ }^{65}$ " n'est pas épargné par cette menace qui est mondiale : «Les forces qui travaillent à la révolution partout dans le monde peuvent fort bien profiter de notre situation particulière pour créer ou attiser un foyer de subversion dans notre coin d'Amérique ${ }^{66}$. "L'Europe n'en est pas moins en première ligne avec, comme perspective finale, un « fracas d'apocalypse " marqué par l'effondrement de " notre civilisation » lorsque la « nouvelle invasion des barbares - masses noires encadrées par des masses jaunes - déferlera sur la vieille Europe, en saccageant, en violant et en massacrant $[. . .]^{67}$ ". L'avenir est très sombre et chacun doit prendre ses responsabilités : "Il est temps que l'homme blanc, responsable en proportion de ses dons supérieurs et de ses grandes réalisations, s'interroge et se ressaisisse ${ }^{68}$."

${ }^{63}$ Robert Rumilly, Le problème national des Canadiens français, Montréal, Fides, 1961, p. 142.

${ }^{64}$ Sur ce point, il utilise en particulier Michel de Saint Pierre, son roman Les nouveaux prêtres et son ouvrage L'école de la violence (Ibid., p. 44-45).

${ }^{65}$ Ibid., p. 63.

${ }^{66}$ Ibid., p. 91.

${ }^{67}$ Ibid., p. 18. Dans un entretien avec André Bastien ("Robert Rumilly, Pourquoi êtes-vous raciste?", Photo-Journal, semaine du 3 au 10 novembre 1965, p. 2), ce dernier répond à propos des barbares et du Tiers-Monde craindre « une réédition des invasions mongoles. Mais, au lieu de venir à cheval, ils viendront dans des chars d'assaut".

${ }^{68}$ Ibid., p. 95. 


\section{Conclusion : le sens d'un combat et d'un itinéraire}

Dès sa brochure sur l'épuration, Rumilly a solennisé son intervention publique : "Il y a des heures où ceux qui ont la chance, l'honneur et la responsabilité d'atteindre le public, par la plume ou par la parole, ont le devoir de parler, de crier, au risque de leur tranquillité personnelle ${ }^{69}$. "Quelques années plus tard, le propos est le même : c'est un «cri d'alarme ${ }^{70}$ " et un appel à la mobilisation qu'il a voulu lancer. Dans son premier Cahier, il a rappelé l'importance du "respect des traditions ${ }^{71}$ ». En 1965, l'objectif de Rumilly est toujours net : "Le monde libre doit retrouver son esprit - son instinct - de conservation ${ }^{72}$. " Cette formule de "monde libre ", caractéristique du contexte de guerre froide, traduit, chez Rumilly, un philo-américanisme même s'il dénonce les progressistes américains.

Rumilly justifie continûment son combat en insistant sur les " inimitiés " que ce dernier lui procure et ce qu'il "risque " en le livrant. Il s'en explique en 1959 : «Ce n'est donc pas de gaieté de cœur que je poursuis ce combat. Dois-je l'avouer? J'ai un peu lambiné dans la préparation de ce "Cahier " par une faiblesse qui ressemblait à de la poltronnerie. J'atteins un âge où l'on recherche la paix, où l'on goûte la considération générale. [...] J'ai surmonté ces hésitations par devoir. Non pas que je me croie une mission. Mais chacun doit servir à sa place et avec ses dons. Je crois, en mon âme et conscience, qu'un immense danger d'ordre spirituel menace notre Canada français. " L'essentiel du propos de Rumilly, eu égard à notre angle de lecture, réside ailleurs, dans cette comparaison constante qu'il opère avec la France et qui lui sert à mettre en garde ses amis canadiensfrançais :

\footnotetext{
${ }^{69}$ Rumilly, La vérité sur la Résistance et l'Épuration en France, p. 1.

${ }^{70}$ Rumilly, Mon cahier $n^{\circ} 3$ : les socialistes dominent le réseau gauchiste, p. 124.

${ }^{71}$ Rumilly, Mon cahier $n^{\circ} 1$ : l'infiltration gauchiste au Canada français, p. 147.

${ }^{72}$ Ibid., p. 24.
} 
Je veux dire que les groupes et les personnalités restés fidèles à l'idéal du Canada français doivent se tenir les coudes, se soutenir les uns les autres. La division voire l'émiettement des nationaux français a toujours été leur grande faiblesse. Il pourrait bien en être de même au Canada français. Il ne s'agit pas de fondre les pensées des uns et des autres dans un même moule. Il y a des nuances dans la pensée des gauchistes. Il peut en subsister dans la nôtre. Mais nous avons en commun l'essentiel. Une solidarité profonde doit nous unir, face au danger. Je crois donc qu'au réseau gauchiste il est temps d'opposer un front national, au moins tacite. Les nationalistes de toutes les nuances [il inclut même certains libéraux et les créditistes] doivent comprendre le caractère profondément antinational du gauchisme ${ }^{73}$.

Ces quelques lignes sont fondamentales. Plus que l'homme de l'Action française (on notera que Rumilly n'emploie pas la formule de "compromis nationaliste "), il faut voir en lui le national français désabusé qui, vocabulaire à l'appui ( Front national »), veut prévenir un échec canadien-français comparable à celui des ligues et de leurs suites. S'il est de nationalité canadienne, s'il est profondément attaché au Canada français ou plus exactement à une certaine vision de ce dernier, Robert Rumilly raisonne à partir d'un cadre français. Le maurrassisme en a été le soubassement mais, quoi qu'en aient dit tant Rumilly que Jean-François Nadeau, il est doctrinalement superficiel et perd de son importance à partir des années 1940 où, sur fond de pétainisme et de catholicisme traditionaliste, Rumilly se fait le relais de certaines publications françaises, à commencer par les Écrits de Paris. Est-on encore dans une orbite maurrassienne? Pour Pierre Trépanier, la chose ne fait pas de doute lorsqu'il présente les publications de René Malliavin : "L'ombre lumineuse de Charles Maurras guidait toujours ces écrivains et ces journalistes, qui ne craignaient pas cependant de rénover, dans la fidélité à son esprit, la doctrine du vieux maître. Délesté de son royalisme, le nationalisme intégral

\footnotetext{
${ }^{73}$ Rumilly, Mon cahier $n^{\circ} 3$ : les socialistes dominent le réseau gauchiste, p. 162.
} 
devenait atlantiste et européen ${ }^{74}$. " Cette interprétation paraît difficilement recevable. On remarquera d'abord qu'Écrits de Paris dans sa relation au maurrassisme (à la différence de la Nation française) est d'abord un conservatoire de sa mémoire plutôt qu'un lieu de rénovation : beaucoup d'anciens y racontent leurs souvenirs, en particulier à la fin des années 1960, mais les très rares articles consacrés aux perspectives ouvertes par le maurrassisme le décrivent comme un astre mort en tant que projet politique ${ }^{75}$. On ne saurait par ailleurs balayer comme un "délestage » le royalisme. Ajoutons encore que le programme des Écrits de Paris sur les points évoqués par Pierre Trépanier est violemment combattu par les maurrassiens ès qualités. Faut-il rappeler ici les oppositions entre Rivarol et Aspects de la France sur la Communauté européenne de défense? Le rejet de la construction européenne par l'Action française est une constante. Quant aux noms cités, de Bardèche à Rebatet, soit ils n'ont jamais été maurrassiens, soit ils ont rompu violemment comme Rebatet. Ce ne fut nullement le cas de Rumilly qui s'est retiré et ne prend nullement part aux débats qui agitent la sphère maurrassienne alors que Pierre Boutang ou Pierre Debray publient dans Tradition et Progrès ${ }^{76}$. Rumilly n'est pas un

${ }^{74}$ Pierre Trépanier, "Robert Rumilly et la fondation du Centre d'Information nationale (1956)", Les Cahiers des Dix, n 44 (1989), p. 232.

75 Georges Aimel, "La fin du maurrassisme ", Écrits de Paris, avril 1961, p. 53-64. Georges Aimel conclut en notant : " [...] si le maurrassisme s'est éteint en tant que somme doctrinale, son esprit demeure comme l'élan d'une foi, non plus seulement en la seule entité France, mais en l'ensemble du destin européen et occidental " (p. 64). Et de souligner l'importance de "l'empirisme organisateur" comme méthode critique.

${ }^{76}$ Sur cette dernière, voir Xavier Gélinas, "La droite intellectuelle et la Révolution tranquille : le cas de la revue Tradition et Progrès, 1957-1962 ", The Canadian Historical Review, vol. 77, n 3 (septembre 1996), p. 353-387, et La droite intellectuelle québécoise et la Révolution tranquille, Québec, Les Presses de l'Université Laval, 2007. Le dépouillement de Tradition et Progrès est instructif. Si Boutang publie un article centré sur le Québec mis en relation avec l'Algérie sur fond d'antigaullisme ("La France "heureuse sans Québec" ", Tradition et Progrès, vol. 3, $\mathrm{n}^{\circ} 1$ (hiver 1960)), pour Pierre Debray, il s'agit d'un article reproduit où, avec force arguments, il exécute Madiran au nom du nationalisme intégral (Tradition et Progrès, vol. $1, \mathrm{n}^{\circ} 2$ (juin-juillet-août 1957), notamment p. 44, où il relève : "C'est 
doctrinaire mais un propagandiste qui cherche à fédérer des oppositions. Le Centre d'Information nationale incarne cette volonté et fut bien, comme l'a souligné Pierre Trépanier, autre chose qu'une " chapelle doctrinale ", mais un lieu qui " admettait, favorisait même, dans certaines limites, la coexistence de tendances diverses ${ }^{77}$ \#. Le Centre d'Information nationale ne saurait cependant être considéré comme une forme de réplique du Front national des années 1930, qui a cherché à fédérer une partie des ligues nationalistes françaises de l'entre-deux-guerres, ligues dont on ne trouve pas, quelle que soit l'homologie du vocable, l'équivalent au Canada français ainsi que l'a montré récemment Michel Bock ${ }^{78}$.

Passeur entre la France et le Canada français, Robert Rumilly fut largement reconnu dans son pays d'adoption où ses idées furent à la fois dénoncées ${ }^{79}$, mais aussi largement diffusées et reprises dans certaines revues comme Tradition et Progrès ${ }^{80}$ qui le publie ${ }^{81}$, en fait un de ses mentors ${ }^{82}$ et prend soin de dénoncer « la légende que l'on

aux nationalistes français, aux nationalistes intégraux, que cette revue [Itinéraires] réserve ses coups les plus durs "). Il est important de souligner que la rédaction le reproduit parce qu'il évoque Marcel Clément, mais il aurait été intéressant de connaître la réaction de Rumilly sur le cas Madiran.

77 Trépanier, "Robert Rumilly et la fondation du Centre d'Information nationale (1956)", p. 234.

${ }^{78}$ Voir la contribution de Michel Bock au colloque Le phénomène ligueur en Europe et aux Amériques, organisé à Metz en septembre 2010 et dont les actes doivent paraître en 2011, sous la direction d'Olivier Dard et Nathalie Sévilla.

${ }^{79}$ Pierre Trépanier, "Robert Rumilly, historien engagé ", L'Action nationale, vol. LXXIII, $\mathrm{n}^{\circ} 1$ (septembre 1983), p. 25.

${ }^{80} \mathrm{Il}$ est instructif de relever, outre des encarts publicitaires pour ses brochures, que les thèses de Rumilly sur Vichy et le " gauchisme " sont reprises notamment lorsqu'il s'agit d'analyser la "contre-idéologie " et la "gauche française " (JeanMarcel Deslauriers, "Nos nouveaux maîtres ", Tradition et Progrès, vol. 1, $\mathrm{n}^{\circ} 1$ (mars 1957), p. 7-8).

${ }^{81}$ Robert Rumilly, "Les obstacles à la survivance franco-américaine ", Tradition et Progrès, vol. 3, n 1 (hiver 1960), p. 14-23.

${ }^{82}$ Rumilly est sollicité pour participer à l'" enquête sur le nationalisme canadienfrançais ". La présentation le concernant, signée Albert Roy, indique : "Il est sans contredit l'un des nôtres, et non des moindres " (Tradition et Progrès, vol. 2 (décembre 1958-mars 1959), p. 25). 
crée d'un Robert Rumilly “important des notions françaises au Canada français" "83 " (l'auteur réfere ici aux notions de " droite " et de "gauche "). Qu'en est-il de la France? Dans les années 1950 et 1960, Rumilly ne figure pas parmi les plumes bien connues du combat antisubversif aux côtés de Suzanne Labin ${ }^{84}$ ou de Jacques Ploncard d'Assac. Même aux Écrits de Paris, Rumilly est peu payé en retour. Il n'est pas complètement ignoré mais lorsqu'il est (très rarement) mentionné, c'est d'abord comme historien du Canada. Ainsi, en 1951, André Thérive, dans sa chronique élogieuse sur l'Histoire du Canada, compare-t-il Rumilly à Seignobos et félicite l'auteur de ses chapitres sur le second conflit mondial. En revanche, il ne fait aucune référence à son combat montréalais en faveur des épurés ${ }^{85}$. Il faut attendre 1979 pour que, dans le portrait très avantageux qui lui est consacré, Rumilly soit présenté dans les Écrits de Paris en tenant compte de son itinéraire. L'identité de l'auteur, le Canadien Jean-Marc Brunet, qui a connu Rumilly, n'est évidemment pas étrangère à ce portrait élogieux. L'article rappelle ainsi qu'il fut ligueur de l'Action française (l'épisode Jonnart est mentionné), défenseur du maréchal Pétain et des épurés (mention de l'affaire Bernonville) et adversaire résolu du "gauchisme " au Canada français. La conclusion est laudative : "Resté fidèle aux idées de sa jeunesse, il ne manque pas une occasion à la radio, à la télévision ou ailleurs, de s'affirmer comme un homme de droite, de rappeler son passage à l'Action française et d'exprimer sa fidélité à un combat qui est aussi le nôtre. Comment ne pas nous réjouir aussi que ce Français, dont la monumentale histoire du Canada fait autorité dans l'ancienne Nouvelle-France

${ }^{83}$ Jean-Marcel Deslauriers, "Le dialogue nationaliste ", Tradition et Progrès, vol. 1, $\mathrm{n}^{\circ} 2$ (juillet-août 1957), p. 5.

${ }^{84}$ Cette dernière évoque à l'occasion le Canada, en particulier dans Le petit livre rouge, arme de guerre, Paris, La Table ronde, 1969, p. 128-130. Rumilly est absent du passage, mais il faut rappeler que Suzanne Labin ne donne aucune référence.

${ }^{85}$ André Thérive, "Lectures françaises ", Écrits de Paris, décembre 1951, p. 110112. Signalons qu'Écrits de Paris mentionne son Histoire de Montréal publiée chez Fides (que la revue orthographie Fidsé) sous la rubrique « Livres nouveaux et rééditions " de juin 1971. 
compte aussi depuis des décennies, parmi les fidèles de notre revue ${ }^{86}$ ? " Si certains points de cet éloge sont matériellement exacts, on ne peut que relever son décalage dans le temps et constater qu'il renvoie à une situation qui était celle de Rumilly à la fin des années 1950 et au début des années 1960 et beaucoup moins celle de la fin des années $1970^{87}$. Entre l'extrême droite française et Rumilly, la relation est donc déséquilibrée, car si l'on peut considérer que Rumilly a été un passeur entre la France et le Canada français, le passage s'est effectué, pour l'essentiel, à sens unique.

${ }^{86}$ Jean-Marc Brunet, "Un Français historien du Canada : Robert Rumilly ", Écrits de Paris, janvier 1979, p. 85.

${ }^{87}$ Robert Rumilly est cependant interrogé en 1976, dans le cadre d'une série d'émissions de Radio-Canada, dont une synthèse a été publiée sous l'égide de Mario Cardinal, Vincent Lemieux et Florian Sauvageau, Si l'Union nationale m'était contée (Montréal, Les Éditions du Boréal Express, 1978), où Rumilly, présenté comme " historien " (p. 233), évoque l'opposition à Duplessis, dont les "gauchistes " (p. 233-235), le père Lévesque (p. 241), et l'opinion de Maurice Duplessis sur les intellectuels (p. 244). 